\title{
A PRELIMINARY CHECK-LIST OF BRITISH MARINE ALGAE
}

\author{
By Mary Parke, D.Sc. \\ The Plymouth Laboratory
}

This preliminary list of some of the classes of the algae has been prepared, mainly for the use of British phycologists, to form a basis on which to work for the eventual compilation of a complete check and locality list of all species of British marine algae. The compiler would be grateful for all additions and corrections to this list, and for records of marine species from the algal classes not included in the present list.

The classes of algae treated in this list are the Chlorophyceae ( 53 gen., I 56 spp.), Xanthophyceae (7 gen., I5 spp.), Chrysophyceae (I 5 gen., 22 spp.), Phaeophyceae (96 gen., I99 spp.), Rhodophyceae (I26 gen., 324 spp.), and Cyanophyceae (39 gen., I25 spp.). The genera are listed alphabetically under the family and the species alphabetically under the genus. If a name has been changed from that given in Newton (I93I), the name in Newton is added, in brackets, below. Names preceded by a question mark appear to need revision for nomenclatural or taxonomic reasons. Most of the so-called varieties listed are very probably merely habitat forms of the species, but further investigation is needed before new combinations are made for them. Notes are given at the end of each class, the number in brackets following a name in the list refers to these notes.

The compiler is grateful to Mr R. Ross and Miss Linda Newton, of the British Museum, and to Miss C. I. Dickinson, of the Herbarium, Kew, for their help over a number of problems. She is also grateful to Miss D. Ballantine for her assistance in checking through some of the literature for marine algal records. Sincere thanks are due to Dr P. Kornmann and to Dr C. Bliding for information. The compiler is also most grateful to Mr F. S. Russell, F.R.S., and to Mr G. M. Spooner for their encouragement and for suggestions as to the form the list should take. 


\section{CHLOROPHYCEAE}

\section{VOLVOCALES}

Chlamydomonadaceae

BRACHIOMONAS Bohlin, I897. simplex Hazen

submarina Bohlin

? westiana Pascher

CARTERIA Diesing, I866. excavata Mass. ex Carter

ChLAMydomonas Ehrenberg, I833. brachyura G. S. West microplankton Rke. quadrilobata Carter

Platymonas G. S. West, r9i6. apiculata Butcher contracta Carter tetrathele G. S. West

Polyblepharidaceae

BIPEDINOMONAS Carter, I937. pyriformis Carter rotunda Carter

Dunaliella Teodoresco, 1905. salina (Dunal) Teodor.

HeTEROMASTIX Korshikov, I923. minuta Carter

PyRAMIMONAS Schmarda, I850. (3) angulata Carter grossii Parke obovata Carter octociliata Carter olivacea Carter

\section{Chlorodendraceae}

PRASINOCLADUS Kuckuck, I894. lubricus Kuck.

\section{CHLOROCOCCALES}

Chlorococcaceae

Characium A. Braun, I849.

? marinum Kjellm. (1)

Chlorochytrium Cohn, I872. cohnii Wright dermatocolax Rke. facciolae (Borzi) Bristol immersum Masse inclusum Kjellm. moorei Gardner willei Printz

Codiolum A. Braun, I855. (2) petrocelidis Kuck. ? pusillum (Lyngb.) Kjellm.

SYKIDION Wright, I88I. dyeri Wright

Chlorellaceae

Chlorella Beijerinck, I890. marina Butcher ovalis Butcher salina Butcher stigmatophora Butcher
NANNOCHLORIS Naumann, I9I9. (3) atomus Butcher maculatus Butcher

Trebouxia de Puymaly, I924. humicola (Treboux) Puymaly

\section{ULOTRICHALES}

Ulotrichaceae

STICHOCOCcus Nägeli, I849. cylindricus Butcher

UlOTHRIX Kützing, I833. (4) consociata Wille flacca (Dillw.) Thur. pseudoflacca Wille subflaccida Wille (? implexa Kütz.)

Monostromaceae

Monostroma Thuret, I854. (5) crepidinum Farl. fuscum (Post. et Rupr.) Wittr. (blyttii (Aresch.) Wittr.) grevillei (Thur.) Wittr. oxyspermum (Kütz.) Doty (laceratum Thur.) (latissimum (Kütz.) Wittr.) (orbiculatum Thur.) (quaternarium (Kütz.) Desm.) (wittrockii Born.) undulatum Wittr.

\section{Ulvaceae}

BLIDINGIa Kylin, I947• minima (Näg.) Kylin

(Enteromorpha minima Näg.) (Enteromorpha micrococca Kütz.) CApsosiphon Gobi, I879. (6) fulvescens (Ag.) Setch. et Gardn (C. aureolus (Ag.) Gobi)

ENTEROMORPHA Link, I820. (7) ahlneriana Bliding clathrata (Roth) Grev. compressa (L.) Grev. intestinalis (L.) Link ? lingulata J. Ag. linza (L.) J. Ag. ? marginata J. Ag. prolifera (Müll.) J. Ag. ralfsii $\mathrm{Harv}$

ramulosa (Sm.) Hook. torta (Mert.) Reinb.

? usneoides (Bonnem.) J. Ag.

PERCURSARIA Bory, I823. percursa (Ag.) Bory

ULVA Linnaeus, I753.

? lactuca $\mathrm{L}$

var. ? lactuca

var. ? latissima (L.) D.C. var. ? rigida (Ag.) Le Jol. 


\section{PRASIOLALES}

Prasiolaceae

GAYELLA Kolderup Rosenvinge, I893. polyrhiza $\mathrm{K}$. Rosenv.

Prasiola Agardh, I822. crispa (Lightf.) Menegh. stipitata Suhr

\section{CHAETOPHORALES}

Chaetophoraceae

ACROCHAETE Pringsheim, I862. parasitica Oltm. repens Pringsh.

BOLBOCOLEON Pringsheim, 1862. piliferum Pringsh.

Chaetobolus Kolderup Rosenvinge, 1893.

gibbus K. Rosenv. (8)

ECTOCHAETE (Huber) Wille, 1909. (9) leptochaete (Huber) Wille (Endoderma leptochaete Huber) wittrockii (Wille) Kylin (Endoderma wittrockii Wille)

ENTOCLADIA Reinke, I879. perforans (Huber) Levring viridis $\mathrm{Rke}$.

(Endoderma viride Lagerh.)

EPICLADIA Reinke, I889. flustrae Rke.

(Endoderma flustrae Batt.)

var. flustrae var. ? phillipsii Batt.

OchlochaETE Thwaites ex Harvey, I849. (8)

ferox Huber hystrix Thwaites

PhAEOPHILA Hauck, 1876. dendroides (Crn.) Batt. engleri Rke.

PILINIA Kützing, I843. maritima (Kjellm.) K. Rosenv. rimosa Kütz.

PringSheimiella v. Hoehnel, I920. scutata (Rke.) Marchew. (Pringsheimia scutata Rke.)

Protoderma Kützing emend. Borzi, I895 marinum Rke.

PSEUDOPRINGSHEIMIA Wille, I909. confluens (K. Rosenv.) Wille (Ulvella confluens $\mathrm{K}$. Rosenv.)

fucicola (K. Rosenv.) Wille (10) (Ulvella fucicola $\mathrm{K}$. Rosenv.)

Pseudulvella Wille, Ig09. applanata Setch. et Gardn.

Ulvella Crouan, I859. lens Crn.

\section{Trentepohliaceae}

? Gomontia Bornet et Flahault, I888. (11)

? manxiana Chodat

? polyrhiza (Lagerh.) Born. et Flah.
Tellamia Batters, I895. contorta Batt.

? intricata Batt.

\section{CLADOPHORALES}

Cladophoraceae (12)

ACrosiphonia J. Agardh; sensu Wille, I909.

centralis (Lyngb.) Kjellm. (13)

(Cladophora (Spongomorpha) arcta (Dillw.) Kütz.)

CHAETOMORPHA Kützing, I845.

? aerea (Dillw.) Kütz.

capillaris (Kütz.) Børg. (14)

crassa (Ag.) Kütz.

linum (Müll.) Kütz.

? litorea Cooke melagonium (Web. et Mohr) Kütz.

ClaDOPHORA Kützing, I843.

albida (Huds.) Kütz.

var. albida

var. refracta (Harv.) Thur.

? arctiuscula Kütz.

? balliana Harv.

brownit (Dillw.) Harv.

cornea Kütz.

var. verticillata Kütz.

? corymbifera Kütz. (17)

corynarthra Kütz.

var. spinescens Batt.

expansa (Mert.) Kütz.

? falcata Harv.

? flexuosa (Griff.) Harv.

fracta (Müll.) Kütz. (15)

glaucescens (Griff.) Harv.

gracilis (Griff.) Kütz.

hirta Kütz.

hutchinsiae (Dillw.) Kütz.

var. hutchinsiae

var. distans (Ag.) Kütz. (16)

var. ? divaricata Harv.

macallana Harv.

magdalenae Harv.

pachyderma (Kjellm.) Brand

var. tenuior (Børg.) Brand

pellucida (Huds.) Kütz.

var. pellucida

var. ? comosa Kütz.

var. ? cristata Kütz.

var. ? curvata Kütz.

prolifera (Roth) Kütz.

ramosissima (Drap.) Kütz.

f. humilis (Kütz.) Hamel

(C. neesiorum Kütz. var. humili Batt.)

rectangularis (Griff.) Harv.

f. rectangularis

f. hispida Kütz.

f. horrida Kütz.

f. subnuda Kütz.

? refracta (Roth) Kütz. (17)

repens (J. Ag.) Harv.

rudolphiana (Ag.) Harv. 
Cladophora (cont.)

rupestris (L.) Kütz.

f. rupestris

f. ? distorta (Harv.)

f. nuda (Harv.) Hamel

? sericea (Huds.) Kütz. (18)

sonderi Kütz.

stolonifera (Kjellm.) Batt.

? traillii (Batt.) Batt.

? trichocoma Kütz.

utriculosa Kütz.

var. utriculosa

var. ? diffusa Hauck

RHIzoclonIUM Kützing, I843.

arenosum (Carm.) Kütz.

? hieroglyphicum (Ag.) Kütz.

implexum (Dillw.) Kütz.

? kerneri Stockm.

kochianum Kütz.

riparium (Roth) Harv.

tortuosum (Dillw.) Kütz. (19)

SPONGOMORPHA Kützing, I843.

bombycina (Kjellm.) Wille

lanosa (Roth) Kütz.

var. lanosa

var. ? uncialis (Müll.)

pallida (Kjellm.) Kylin

UROSPORA Areschoug, I866.

bangioides (Harv.) Holm. et Batt.

penicilliformis (Roth) Aresch. (2) (isogona (Sm.) Batt.)

sporophyte Codiolumgregarium $\mathrm{A} . \mathrm{Br}$. speciosa (Carm.) Leblond ex Hamel (Ulothrix speciosa (Carm.) Kütz.)

wormskioldii (Mert.) K. Rosenv. (collabens (Ag.) Holm. et Batt.)
? SIPHONALES

Caulerpaceae

BRYOPSIS Lamouroux, I809.

hypnoides Lamour.

plumosa (Huds.) Ag.

var. plumosa

var. ? nuda Holm.

var. ? subsimplex Holm. et Batt.

\section{Derbesiaceae}

DERBESIA Solier, I847. (20)

marina (Lyngb.) Kjellm.

gametophyte $=$ Halicystis ovalis

(Lyngb.) Aresch.

tenuissima (De Not.) Crn.

\section{Codiaceae}

Codium Stackhouse, 1797.

adhaerens (Cabr.) Ag.

? amphibium Moore

bursa (L.) Ag.

? fragile (Sur.) Hariot

f. atlanticum (Cotton) Levring

(tomentosum var. atlanticum Cotton) ? tomentosum Stackh.

\section{Chaetosiphonaceae}

BLASTOPHYSA Reinke, I888. rhizopus Rke.

\section{Phyllosiphonaceae}

OSTREOBIUM Bornet et Flahault, I889. quekettii Born. et Flah.

\section{Notes on Chlorophyceae}

(1) Not an independent species according to Levring (1937)—a Cladophora sporeling.

(2) C. gregarium A.Br., diploid generation of Urospora penicilliformis; C. petrocelidis, probably an independent form.

(3) Systematic position uncertain; see Chadefaud (I94I) on Pyramimonas Schmarda.

(4) British species of Ulothrix require revision.

(5) British species in urgent need of revision: see Hamel (I93I) for species included under M. oxycoccum (Kütz.) Thur. =M. oxyspermum (Kütz.) Doty.

(6) Chapman (1952) advocates the removal of the genus Capsosiphon from the Ulvaceae to separate family, Capsosiphonaceae.

(7) Until British material belonging to this genus has been fully investigated on the lines of the work of Bliding, the limits of the species occurring in Britain cannot be assessed.

Dr C. Bliding has checked the list of species personally, and has very kindly allowed me to publish his records of $E$. ahlneriana for the British coast. He reports:

'During an excursion to Anglesey, North Wales, in July 1953, I found Enteromorpha Ahlneriana Bliding in the intertidal zone at two different localities, Rhosneigr and Newborough.

'At Rhosneigr the main axes of the thalli were often very broad with few branches, as in Typus III (Bliding, 1944, p. 343, fig. I6). The material from Newborough, however, was more branched and the axes of the plants, as well as the branches, were more slender.

' $E$. Ahlneriana has so far been recorded only from the west coast of Sweden and from the Baltic Sea. In the Baltic this species is very abundant.' (Carl Bliding, Plymouth, 17 July I953.) 
(8) Chaetobolus gibbus, Ochlochaete ferox and O. hystrix-possibly forms of one species, see Waern (1952).

(9) Endoderma-Ectochaete-Entocladia-Epicladia complex-British material needs revision.

(10) Ulvella fucicola var. globosa Batt.; before making new combination under Pseudopringsheimia further investigation seems desirable.

(11) If Kylin (1935) is right Gomontia is a name of a taxon derived from two entirely discordant elements and should perhaps be rejected (Art. 76, Lanjouw, 1952).

(12) The British species of all genera in this family are in urgent need of revision.

(13) Part at least, if not all, material recorded for Britain under $\mathrm{Cl}$. (Spongomorpha) arcta should be referred to Acrosiphonia centralis (Lyngb.) Kjellm.

(14) ='Conferva tortuosa J. Ag.', Harv., Phyc. Brit. 54A, Chaetomorpha tortuosa Kütz., in Newton, I93I, p. 9I (Chapman, 1939).

(15) British forms referred to this species are in need of revision.

(16) It is generally considered that the alga described and figured by Harvey, Phyc. Brit., I30, as Cladophora diffusa is C. hutchinsiae Kütz. var. distans (Ag.) Kütz., which differs from Conferva diffusa Roth (=Cladophora diffusa (Roth) Harv.).

(17) Possibly forms of Cladophora hamosa Kütz.

(18) Should possibly be Cladophora crystallina (Roth) Kütz., see Levring (1937, I940), Waern, 1952.

(19) $=$ Conferva tortuosa Dillw., Conferva implexa Harv., Phyc. Brit., 54B, Lola implexa (Harv.) Hamel (Chapman, 1939).

(20) HALICYSTIS Areschoug, I850; gametophyte generation of Derbesia marina (Lyngb.) Kjellm. (Halicystis ovalis (Lyngb.) Aresch.) recorded for Britain but not the gametophyte generation (H. parvula Schmitz) of Derbesia tenuissima (De Not.) Crn.

\section{XANTHOPHYCEAE}
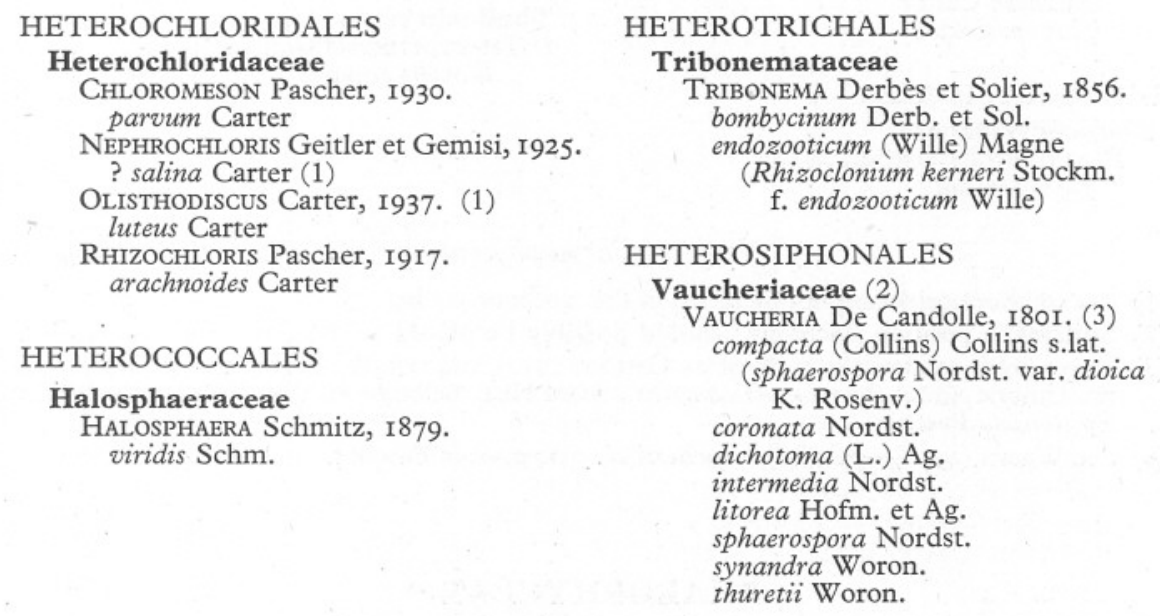

\section{Notes on Xanthophyceae}

(1) Systematic position uncertain.

(2) Reasons for placing in Xanthophyceae-see Chadefaud (1945, I95I), Smith (I950).

(3) The list of species given has been checked by Mr T. Christensen, who also says that the Vaucheria sp. recorded by Carter as possibly $V$. woroniniana Heer. is definitely not this species. See also Christensen (1952) for information on distribution. 


\section{CHRYSOPHYCEAE}

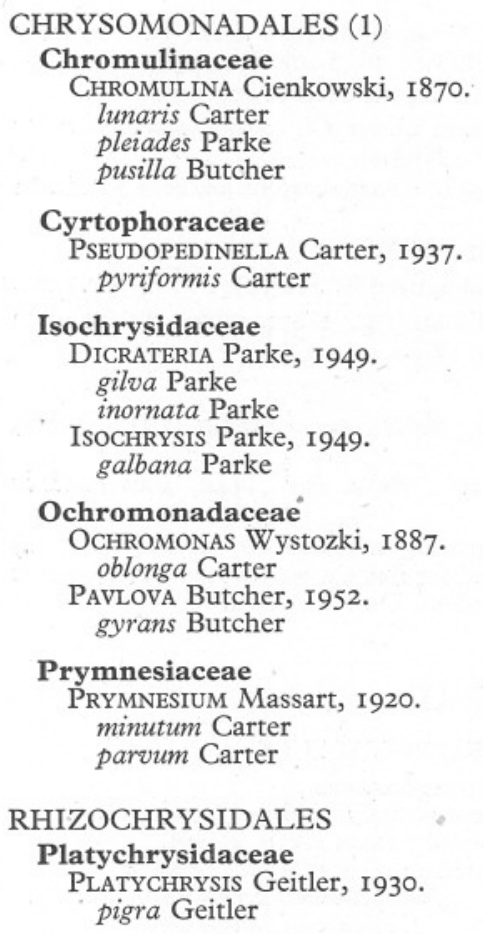

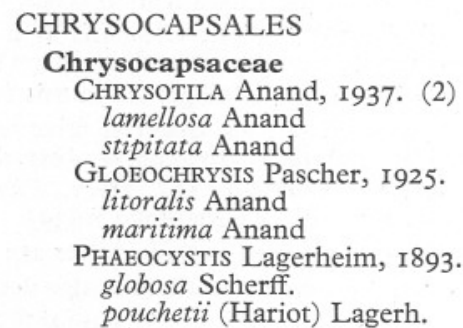

\section{CHRYSOTRICHALES}

Nematochrysidaceae

NeMATOCHRYsIS Pascher, I925. sessilis Pascher var. vectensis Carter

\section{Phaeothamnionaceae}

APISTONEMA Pascher, I925. (3) carteri Anand (4)

Chrysonema Anand, I937. litorale Anand

Thallochrysidaceae

THALlOCHRYSIS Conrad, I920. litoralis Anand

\section{Notes on Chrysophyceae}

(1) Coccolithophoridaceae not included in this preliminary list.

(2) Systematic position uncertain: should possibly be placed as the type of a new family.

(3) Phaeococcus adnatus G. S. West ex Cotton (I9I2), imperfectly described by Cooke and Rabenhorst under the name Gloeocystis adnata Näg., belongs, very possibly, to the genus Apistonema Pascher.

(4) See Waern (1952)-possibly a form of A. pyrenigerum Pascher.

\section{PHAEOPHYCEAE}

\section{ECTOCARPALES}

Ectocarpaceae (1)

ACINETOSPORA Bornet, I89I. crinita (Carm.) Kornmann (2) (pusilla (Griff.) Born.) (pusilla var. crinita (Carm.) Batt.) haploid generation $=$

? Ectocarpus lebelii Crn.

? Ectocarpus padinae Sauv.
Chilionema Sauvageau, i897. (3) ocellatum (Kütz.) Kuck. (nathaliae Sauv.) (ocellatum (Rke.) Sauv.) reptans (Crn.) Sauv.

Compsonema Kuckuck, I899. (3) microspongium (Batt.) Kuck. (Ectocarpus microspongium Batt.) saxicola Kuck. (Myrionema saxicolum Kuck.) 
? DichosporangIUM Hauck, I885. ? chordariae Wollny (4)

(Streblonema chordariae Cotton in Newton, p. I28, non S. chordariae (Farl.) De Toni)

ECTOCARPUS Lyngbye, I8I9.

? acanthophorus Kütz.

confervoides (Roth) Le Jol. s. lat. (confervoides s.str.)

(amphibius Harv.)

(arctus Kütz.)

(dasycarpus Kuck.)

(hiemalis Crn.)

(penicillatus (Ag.) Kjellm.)

(siliculosus (Dillw.) Lyngb.)

congestus Crn.

crouanii Thur.

? distortus Carm.

draparnaldioides (Crn.) Kjellm.

? erectus Kütz.

fasciculatus Harv.

? fenestratus Berk. ex Harv.

holmesii Batt:

? landsburgii Harv.

? minimus Näg. (5)

reinboldii Rke.

ENDODICTYON Gran, I897.

infestans Gran (9)

(Streblonema infestans Batt.)

Feldmannia Hamel, i939.

battersii (Born.) Hamel (Ectocarpus battersii Born.)

globifera (Kütz.) Hamel (Ectocarpus globifer Kütz.) (6)

? irregularis (Kütz.) Hamel (Ectocarpus irregularis Kütz.)

simplex (Crn.) Hamel

(Ectocarpus simplex Crn.)

GifFordia Batters emend. Hamel, I939.

granulosa (Sm.) Hamel

(Ectocarpus granulosus (Sm.) Ag.)

hincksiae (Harv.) Hamel (Ectocarpus hincksiae Harv.)

mitchellae (Harv.) Hamel (Ectocarpus mitchellae Harv.) ovata (Kjellm.) Kylin

(Ectocarpus ovatus Kjellm.) var. ovata

var. arachnoidea (Rke.) Kylin

sandriana (Zanard.) Hamel (Ectocarpus sandrianus Zanard.)

secunda (Kütz.) Batt.

(Ectocarpus secundus Kütz.)

HeCATONEMA Sauvageau 1897. (3)

liechtensternii (Hauck) Batt.

maculans (Coll.) Sauv.

speciosum (Børg.) Cotton

HeRponema J. Agardh emend. Hamel, I939.

luteolum (Sauv.) Hamel

(Ectocarpus luteolus Sauv.)

solitarium (Sauv.) Hamel

(Ectocarpus solitarius Sauv.) valiantei (Born.) Hamel

(Ectocarpus valiantei Born.)

velutinum (Grev.) J. Ag.

(Ectocarpus velutinus (Grev.)

Kütz.) (7)

LAMINARIOCOLAX Kylin, I947.

tomentosoides (Farl.) Kylin

(Ectocarpus tomentosoides Farl.) (8)

MikROSYPHAR Kuckuck, I895. (9) polysiphoniae Kuck.

porphyrae Kuck.

PhaeOSTROMa Kuckuck, I895. pustulosum Kuck. (10)

(P. prostratum Kuck.)

PyLAIELLA Bory, I823.

littoralis (L.) Kjellm. (11)

SOROCARPUS Pringsheim, I862. micromorus (Bory) Silva (uvaeformis (Lyngb.) Pringsh.)

SPONGONEMA Kützing, I849. tomentosum (Huds.) Kütz. (Ectocarpus tomentosus (Huds.) Lyngb. E. terminalis Kutz.) (3)

Streblonema Derbès et Solier, I85I. (12)

? aequale Oltm. (10)

breve (Sauv.) De Toni

(Ectocarpus brevis Sauv.)

effusum Kylin

fasciculatum Thur.

var. fasciculatum

var. ? simplex Batt.

? helophorum (K. Rosenv.) Batt. intestinum (Reinsch) Holm. et Batt. parasiticum (Sauv.) De Toni

(Ectocarpus parasiticus Sauv.) sphaericum (Derb. et Sol.) Thur.

stilophorae (Crn.) De Toni

(Ectocarpus stilophorae Crn.) (13)

var. stilophorae

var. caespitosum (K. Rosenv.)

De Toni

? tenuissimum Hauck (13)

volubile (Crn.) Thur.

zanardinii (Crn.) De Toni

WAERNIELLA Kylin, I947. lucifuga (Kuck.) Kylin

\section{Lithodermataceae}

LITHODERMA Areschoug, I875. extensum (Crn.) Hamel (14) (fatiscens Aresch. emend. Kuck.)

Petroderma Kuckuck, I897. maculiforme (Wollny) Kuck.

SORAPION Kuckuck, I894. simulans Kuck.

\section{Ralfsiaceae}

RALFSIA Berkeley, I83I. (15)

clavata (Carm.) Farlow

disciformis Crn.

pusilla (Strömf.) Fosl.

spongiocarpa Batt. (16)

verrucosa (Aresch.) J. Ag. 
Myrionemataceae

AsCOCYCLUS Magnus, 1874. foecundus (Strömf.) Cotton hispanicus Sauv. magni Sauv. (orbicularis Magn. in Newton, p. I59)

MICROSPONGIUM Reinke, I888. globosum Rke.

(Hecatonema globosum (Rke.) Batt.)

MyrionEMA Greville, I827. aecidioides (K. Rosenv.) Sauv. (12) corunnae Sauv. papillosum Sauv. polycladum Sauv. strangulans Grev.

SYMPHYOCARPUS Kolderup Rosenvinge, I894. strangulans $\mathrm{K}$. Rosenv.

? Ulonema Foslie, I894. (17) ? rhizophorum Fosl.

\section{Elachistaceae}

Elachista Duby, I830. (18) flaccida (Dillw.) Aresch. fucicola (Vell.) Aresch.

f. fucicola

f. grevillei (Arnott) Hamel (E. grevillei Arnott) scutulata (Sm.) Duby

HALOTHRIX Reinke, I888. lumbricalis (Kütz.) Rke.

LEPTONEMA Reinke, I888. fasciculatum Rke.

var. fasciculatum

var. ? majus Rke. (19)

var. ? subcylindrica K. Rosenv. (19)

var. ? uncinatum Rke. (19)

SyMPHORICOCCUS Reinke, I888. stellaris (Aresch.) Kuck. (20) (Elachista stellaris Aresch.)

\section{Leathesiaceae}

CORYNOPHLAEA Kützing, I843. crispa (Harv.) Kuck. (Leathesia crispa Harv.)

CYLINDROCARPUS Crouan, I85I. (Petrospongium Näg.)

berkeleyi (Grev.) Crn. (Petrospongium berkeleyi Näg.) microscopicus Crn.

(Ectocarpus microscopicus Batt.)

LEATHESIA S. F. Gray, I82I. difformis (L.) Aresch.

MiCROCORYNE Strömfelt, I 888. ocellata Strömf. (21)

Myriactula Kuntze, I898. (Myriactis Kütz.) areschougii (Crn.) Hamel chordae (Aresch.) Levring (Elachista stellaris var. chordae Aresch.)

clandestina (Crn.) J. Feldm.

(Ectocarpus clandestinus (Crn.) Sauv.) haydenii (Gatty) Levring rivulariae (Suhr) J. Feldm. (Myriactis pulvinata Kütz.) stellulata (Griff.) Levring
Mesogloiaceae

CHORDARIA Agardh emend. Lyngbye, I8I9.

flagelliformis (Müll.) Ag.

var. flagelliformis var. ? firmus Kjellm.

var. ? minor J. Ag.

Cladosiphon Kützing, I843. contortus (Thur.) Kylin (Castagnea contorta Thur.) zosterae (J. Ag.) Kylin (Castagnea zosterae (J. Ag.) Thur.)

EUDESME J. Agardh, I880. virescens (Carm.) J. Ag. (Castagnea virescens (Carm.) Thur.)

LIEBMANNIA J. Agardh, I842. leveillei J. Ag. (Mesogloia leveillei Menegh.)

Mesogloia Agardh, I8I7. lanosa Crn. ? neglecta Batt. (22) vermiculata (Sm.) Le Jol.

MYRIOCLADIA J. Agardh, I84I. (23) tomentosa Crn.

SAUVAGEAUGLOIA Hamel, I939. griffithsiana (Grev.) Hamel (Mesogloia griffithsiana Grev.)

SPHAEROTRICHIA Kylin, I940. divaricata (Ag.) Kylin (Chordaria divaricata Ag.)

STREPSITHALIA Sauvageau, I896. buffhamiana (Batt.) Batt.

Acrothricaceae

ACROTHRIX Kylin, I907. gracilis Kylin

Spermatochnaceae

SPERMATOCHNUS Kutzing emend. Reinke, 1889. paradoxus (Roth) Kütz.

STILOPHORA J. Agardh, I84I. rhizodes (Ehrh.) J. Ag. tuberculosa (Horn.) Rke.

STILOPSIS Kuckuck, I929. lejolisii (Thur.) Kuck. (Spermatochnus lejolisii Rke.)

\section{DICTYOSIPHONALES}

\section{Striariaceae}

ISTHMOPLEA Kjellman, I877. sphaerophora (Carm.) Kjellm.

STICTYOSIPHON Kützing, I843. griffithsianus (Le Jol.) Holm. et Batt. (24)

(Phloeospora brachiata Born. in Newton, p. I87) soriferus (Rke.) K. Rosenv. (25) subarticulatus (Aresch.) Hauck (25) tortilis (Rupr.) Rke. (25)

STRIARIA Greville, I829. attenuata Grev.

f. attenuata

f. crinita (Ruch.) Hauck 


\section{Myriotrichiaceae}

BuFFHAMIA Batters, I895. (26) speciosa Batt.

LEBLONDIELLA Hamel, I939. densa (Batt.) Hamel (Myriotrichia densa Batt.)

MYRIOTRICHIA Harvey, I834. clavaeformis Harv.

var. clavaeformis

var. ? minima Holm. et Batt. ? filiformis Harv. (27)

repens (Hauck) Karsakoff

\section{Giraudiaceae}

Giraudia Derbès et Solier, I85 I. (26) sphacelarioides Derb. et Sol.

\section{Punctariaceae}

DESMOTRICHUM Kützing, I845. undulatum (J. Ag.) Rke. (balticum Kütz.) (28) (Punctaria tenuissima Grev.)

LitosipHON Harvey, I849. filiformis (Rke.) Batt. var. filiformis var. ? gracilis Batt. laminariae (Lyngb.) Harv. (hibernicus (Johns.) Batt.) pusillus (Carm.) Harv.

Punctaria Greville, I830. (29) crispata (Kütz.) Batt.

latifolia Grev. var. latifolia var. ? laminarioides Holm. et Batt. var. ? lanceolata Batt. plantaginea Grev. var. plantaginea var. ? crouanii Thur. var. ? rubescens Batt.

\section{Asperococcaceae}

ÂSPEROCOCCUS Lamouroux, I8I3. bullosus Lamour.

compressus Griff. ex Hook. (30) fistulosus (Huds.) Hook.

f. fistulosus

f. vermicularis (Griff.) Harv. scaber Kuck.

\section{Dictyosiphonaceae}

DictYosIPHON Greville, I830. (31) chordaria Aresch. (mesogloia Aresch.)

(Gobia baltica Rke.)

ekmanii Aresch.

foeniculaceus (Huds.) Grev.

f. foeniculaceus

f. hippuroides (Lyngb.) Levring

(D. hippuroides Kütz.)

\section{SCYTOSIPHONALES (32) \\ Phaeosaccionaceae \\ PhAEOSACCION Farlow, I882. collinsii Farl.}

\section{Scytosiphonaceae}

Colpomenia Derbès et Solier, I856. peregrina Sauv.

Petalonia Derbès et Solier, I850. (33) fascia (Müll.) Kuntze (34) (debilis (Ag.) Derb. et Sol.) (Phyllitis fascia Kütz.) ? filiformis (Batt.) zosterifolia (Rke.) Kuntze (Phyllitis zosterifolia Rke.)

SCytosiphoN Agardh, I8II. lomentaria (Lyngb.) Endl. (pygmaeus Rke.)

var. lomentaria var. ? zostericola Thur.

\section{TILOPTERIDALES \\ Tilopteridaceae \\ HAPLOSPORA Kjellman, I872. globosa Kjellm. \\ TILOPTERIS Kützing, I849. mertensii (Sm.) Kütz.}

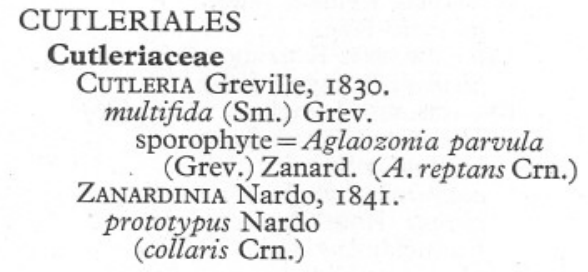

\section{SPOROCHNALES}

Sporochnaceae

CARPOMITRA Kützing, I843. costata (Stackh.) Batt.

SPOROCHNUS Agardh, I8I7. pedunculatus (Huds.) Ag.

\author{
DESMARESTIALES \\ Arthrocladiaceae \\ ARTHROCladia Duby, I832. \\ villosa (Huds.) Duby

\section{Desmarestiaceae} \\ DESMARESTIA Lamouroux, I8I3. \\ aculeata (L.) Lamour. \\ dudresnayi Lamour. \\ ligulata (Lightf.) Lamour. \\ var. ligulata \\ var. ? angustior Batt. \\ var. ? dilatata Batt. \\ viridis (Müll.) Lamour.
}




\section{LAMINARIALES}

\section{Chordaceae}

CHORDA Stackhouse, I797. (35) filum (L.) Stackh. tomentosa Lyngb.

\section{Laminariaceae}

LAMINARIA Lamouroux, I8I3. digitata (Huds.) Lamour. hyperborea (Gunn.) Fosl. (cloustonii Edm.) ochroleuca La Pylaie saccharina (L.) Lamour. (hieroglyphica J. Ag.)

SACCORHIZA La Pylaie, I829. polyschides (Lightf.) Batt. (bulbosa (Huds.) La Pylaie)

\section{Alariaceae}

ALARIA Greville, I830. esculenta (L.) Grev.

\section{SPHACELARIALES}

Choristocarpaceae

CHORISTOCARPUS Zanardini, I860. tenellus (Kütz.) Zanard.

\section{Sphacelariaceae}

BATTERSIA Reinke, I89o. mirabilis Rke.

ChaETOPTERIS Kützing, I843. plumosa (Lyngb.) Kütz.

SPHACELARIA Lyngbye, I8I9. (36) bipinnata (Kütz.) Sauv. britannica Sauv. caespitula Lyngb. cirrosa (Roth) Ag. fusca (Huds.) Ag. plumigera Holm. plumula Zanard. racemosa Grev. radicans (Dillw.) Ag. ? saxatilis (Kuck.) Sauv. tribuloides Menegh.

SPHACELLA Reinke, I890. subtilissima Rke.

\section{Stypocaulaceae}

HALOPTERIS Kützing emend. Sauvageau, 1903 . filicina (Grat.) Kütz. var. filicina var. patentissima Sauv. (sertularia Batt.)

scoparia (L.) Sauv. var. scoparia var. patentissima Sauv. (scoparioides Holm. et Batt.)
DICTYOTALES

\section{Dictyotaceae}

DICTYOPTERIS Lamouroux, I809. membranacea (Stackh.) Batt.

DictYota Lamouroux, I809. dichotoma (Huds.) Lamour. var. dichotoma var. intricata (Ag.) Grev. var. ? latifrons Holm. et Batt. ILOPHUS J. Agardh, I880. spiralis (Mont.) Hamel (Dictyota ligulata Kütz.)

Padina Adanson, I763. pavonia (L.) Lamour.

TAONIA J. Agardh, I848. atomaria (Woodw.) J. Ag. var. atomaria var. ? divaricata Holm. et Batt.

\section{FUCALES}

Fucaceae

AsCOPHYLluM Stackhouse, I809. nodosum (L.) Le Jol.

f. nodosum

f. mackaii (Turn.)

(A. mackaii (Turn.) Holm. et Batt.)

f. scorpioides (Hornem.) Hauck

Fucus Linnaeus, I753. (37) ceranoides $\mathrm{L}$. inflatus L. (anceps Harv. et Ward.) serratus $\mathrm{L}$. spiralis L. vesiculosus $\mathrm{L}$.

Pelvetia Decaisne et Thuret, I845. canaliculata (L.) Dcne. et Thur.

\section{Himanthaliaceae}

Himanthalia Lyngbye, I8I9. elongata (L.) S. F. Gray (lorea (L.) Lyngb.)

\section{Cystoseiraceae}

BIFURCARIA Stackhouse, I809. rotunda (Huds.) Papenf. (tuberculata (Huds.) Stackh.)

Cystoseira Agardh, I820. fibrosa (Huds.) Ag. foeniculacea (L.) Grev. emend. Sauv. (discors Ag.) granulata Ag. tamariscifolia (Huds.) Papenf. (ericoides (L.) Ag.)

Halidrys Lyngbye, I8I9. siliquosa (L.) Lyngb.

\section{Cladostephaceae}

Cladostephus Agardh, I8I7. spongiosus (Huds.) Ag. verticillatus (Lightf.) Ag. 


\section{Notes on Phaeophyceae}

(1) British material belonging to genera in this family is in urgent need of revision.

(2) See Kornmann (1953): Dr Kornmann very kindly sent me proofs of his paper and also of that of Kuckuck (1953), so that the information contained in these papers could be included here.

(3) See Kuckuck (1953).

(4) The genus Dichosporangium is included here for the present to cover Streblonema chordariae Cotton in Newton (I93I). If remaining in Streblonema it would need a new name because of earlier $S$. chordariae (Farl.) De Toni.

(5) Requires investigation. Hamel (1939), suggests it should be placed in Herponema J. Ag. emend. Hamel.

(6) New combination not made for var. rupestris Batt., investigation needed.

(7) New combination not made for var. laterifructus Batt., investigation needed.

(8) New combination not made for var. punctiformis Batt., investigation needed.

(9) See Levring (1945) and Waern (1952).

(10) Jaasund (I95I) considers genus should be placed in the Chordariaceae; also considers that Streblonema aequale Oltm. is possibly a form of Phaestroma pustulosum. According to Cotton (1912) British material differs slightly from Kuckuck's plant and may possibly be a distinct species.

(11) Concerning validity of name and also Pylaiella rupincola (Aresch.) Kylin—see information given in Waern (1952).

(12) Streblonema s.lat.; British material belonging to this complex in urgent need of investigation and revision, see Hamel (1939), Kylin (1947), Waern (1952). British species placed by Hamel (1939) in Entonema Reinsch sensu Hamel (Streblonema parasiticum (Sauv.) De Toni, S. breve (Sauv.) De Toni, Ectocarpus clandestimus (Crn.) Sauv.) and by Kylin (1947) in Entonema Reinsch sensu Kylin (Streblonema effusum Kylin, S. aequale Oltm., Myrionema aecidioides (K. Rosenv.) Sauv.) have, for the time being, been left, either in the genus Streblonema s.lat., or in other genera in which they have been placed.

(13) Ectocarpus stilophorae Crn. var. cervicornis Batt. recorded, investigation needed. Is Streblonema tenuissimum Hauck a form of S. stilophorae (Crn.) De Toni?

(14) Almost certainly more than one species recorded for British coast under name $L$. fatiscens Aresch., see Waern (1952). British material of whole family in need of investigation.

(15) Genus in need of revision, see Hamel (1939), Hollenberg (1941), Kylin (1947).

(16) According to Batters $=R$. clavata Rke. non Farlow; see Kylin (1947) who gives new name $R$. tenuis for Reinke's $R$. clavata; requires investigation.

(17) See Cotton (I9I2), Levring (1937), Kylin (1947), Jaasund (I95I).

(18) Conserved as Elachista, original spelling Elachistea, which is etymologically incorrect according to Fritsch (1945).

(19) Habitat forms of species?

(20) = Areschougia stellaris (Aresch.) Menegh. See Silva (1952), where Areschougia Harv. is proposed for conservation against Areschougia Menegh.

(21) See Hamel (1939).

(22) See Batters (I906), and Cotton (I9I2, p. i24, under Acrothrix.)

(23) According to Kylin (1933) the plant recorded on the British coast under the name Myriocladia lovenii is not the true $M$. lovenii J. Ag.; it may be an undescribed species.

(24) The following notes are from Mr R. Ross and Miss L. Newton concerning the confusion that has been found in the synonomy dealing with this plant. The type specimen of Conferva brachiata Sm. in Engl. Bot. has been examined and is thought to be a Pylaiella. This specimen is also the nomenclatural type of Ectocarpus brachiatus (Sm.) Harv. ex Hook. non C. Ag., Phloeospora brachiata (Sm.) Bornet, Stictyosiphon brachiatus (Sm.) Hygen et Jorde. The plant found by Mrs Griffiths and described and figured under the above names by all authors is quite different. Its earliest legitimate name is Ectocarpus griffithsianus Le Jolis in Trans. bot. Soc. Edinb., vol. 7, p. 37 (1863), and a combination based on this name in the appropriate genus is its correct name. The plant has been left for the time being in the genus Stictyosiphon, but further study is needed.

(25) British material in urgent need of investigation; kept as three separate species for the present, but see Kolderup Rosenvinge (1935), Levring (1937, 1940), Kolderup Rosenvinge \& Lund (1947), Waern (1952).

(26) Systematic position uncertain. 
(27) British material needs investigation; should be perhaps $M$. clavaeformis f. filiformis (Harv.) Kjellm., see Kolderup Rosenvinge \& Lund (1947), Kylin (1947).

(28) See Kylin (1947), Kolderup Rosenvinge \& Lund' 1947).

(29) British material needs study, see Hamel (1939), Kolderup Rosenvinge \& Lund (1947).

(30) See Hamel (1939), who places this species in genus Haloglossum Kütz.

(31) British material of genus in need of investigation; see Du Rietz (1940), Levring (1940), Kolderup Rosenvinge \& Lund (1947), Sinclair (1949).

(32) See J. Feldmann (I949).

(33) See Silva (1952) concerning name of genus.

(34) See Setchell \& Gardner (1925) under Ilea Fries; field observations needed before British forms of species can be revised and the limits of the species assessed.

(35) Recorded varieties of both species require investigation before being listed. Kolderup Rosenvinge \& Lund (1947) state that the var. subtomentosa of Chorda filum is not to be maintained.

(36) British material of genus in need of revision, see Waern (1945, 1952), Kylin (1947), Lund (1950).

(37) No varieties or forms given in list; awaiting the results of Dr E. Burrows and Dr S. Lodge's work on the form-range of the Fucus species (except F. inflatus) on the British coast. $\mathrm{Mr} \mathrm{H}$. Powell is investigating $F$. inflatus $\mathrm{L}$.

\section{RHODOPHYCEAE}

\section{BANGIOPHYCIDAE \\ GONIOTRICHALES \\ Goniotrichaceae \\ AsTEROCYTIS Gobi, I879. ramosa (Thwaites) Gobi (1) \\ GoNIOTRICHUM Kützing, I843. ? elegans (Chauv.) Le Jol. cornu-cervi (Reinsch) Hauck \\ NeEvEA Batters, I900. repens Batt.}

\section{BANGIALES}

Erythropeltidaceae

ERYTHROCLADIA Kolderup Rosenvinge, I909.

irregularis $\mathrm{K}$. Rosenv.

subintegra $\mathrm{K}$. Rosenv.

ERYTHROPELTIS Schmitz, I896. (2)

? discigera Schm.

var. ? discigera

var. ? flustrae Batt.

ERYTHROTRICHIA Areschoug, I850. (3) bertholdii Batt.

boryana (Mont.) Berth.

var. boryana

var. ? crispa Batt. carnea (Dillw.) J. Ag.

ciliaris (Carm.) Batt. investiens (Zanard.) Born. reflexa (Crn.) Thur. welwitschii (Rupr.) Batt.

PORPHYROPSIS Kolderup Rosenvinge, I909.

coccinea (J. Ag.) K. Rosenv.

\author{
Bangiaceae \\ BANGIA Lyngbye, I819. \\ fuscopurpurea (Dillw.) Lyngb. (4) \\ CoNCHOCELIS Batters, I892. (5) \\ ? rosea Batt. \\ PORPHYRA Agardh, I824. \\ amethystea Kütz. \\ leucosticta Thur. \\ linearis Grev. \\ miniata (Lyngb.) Ag. \\ f. miniata \\ f. abyssicola (Kjellm.) K. Rosenv. \\ f. amplissima (Kjellm.) K. Rosenv. \\ f. tenuissima (Strömf.) K. Rosenv. \\ umbilicalis (L.) Kütz. \\ f. umbilicalis \\ f. laciniata (Lightf.) J. Ag.
}

\section{FLORIDEOPHYCIDAE \\ NEMALIONALES}

Acrochaetiaceae

ACROCHAETIUM Nägeli, r86r. (6)

alariae (Jonss.) Born.

battersianum Hamel

bonnemaisoniae (Batt.) J. et G. Feldm. (Colaconema bonnemaisoniae Batt.)

bornetii Papenf. (corymbiferum (Thur.) Batt.)

brebneri (Batt.) Hamel

(Rhodochorton brebneri Batt.)

caespitosum (J. Ag.) Näg.

? (lorrain-smithiae (Lyle) Newton)

chylocladiae (Batt.) Batt.

f. chylocladiae

f. pulchrum Batt.

daviesii (Dillw.) Näg. 
ACROCHAETIUM (cont.)

emergens (K. Rosenv.) Web.-v. Bosse

endophyticum (Batt.) Batt.

endozoicum (Darb.) Batt.

microscopicum (Näg.) Näg.

minutum (Suhr.) Hamel

(Rhodochorton minutum (Suhr) Rke.)

mirabile Näg.

nemalionalis (De Not.) Born.

pallens (Zanard.) Näg.

(Rhodochorton pallens (Zanard.) Hauck)

parvulum (Kylin) Hoyt

reticulatum (Batt.) Papenf.

(Colaconema reticulatum Batt.)

sanctae-mariae (Darb.) Hamel

scapae (Lyle) Papenf.

secundatum (Lyngb.) Näg.

seiriolanum (Harvey-Gibs.) K. Rosenv.

sparsum (Harv.) Näg.

thuretii (Born.) Collins et Hervey

trifilum (Buff.) Batt.

virgatulum (Harv.) J. Ag.

f. virgatulum

f. luxurians (J. Ag.)

f. tetrica (K. Rosenv.)

? griffithsianum Näg.

? irregulare Reinsch

? lanuginosum (Dillw.) Näg.

? pulvereum Näg.

Audouinella Bory emend. Papenfuss, I945.

efflorescens (J. Ag.) Papenf.

(Acrochaetium efflorescens Näg.)

membranacea (Magn.) Papenf.

(Rhodochortonmembranaceum Magn.)

RHODOCHORTON Nägeli, I86I. (7)

Helminthocladiaceae

Helminthocladia J. Agardh, I85I. calvadosii (Lamour.) Setch. (purpurea J. Ag.)

? hudsonii J. Ag.

Helminthora J. Agardh, I85I. divaricata (Ag.) J. Ag.

Nemalion Duby, I830. elminthoides (Vell.) Batt. ? multifidum (Web. et Mohr) J. Ag.

\section{Chaetangiaceae}

ScINAIA Bivona, I822.

furcellata (Turn.) Bivona

subcostata (J. Ag.) Chemin ex Hamel (furcellata var. subcostata J. Ag.

\section{GELIDIALES \\ Gelidiaceae \\ Pterocladia J. Agardh, I852. pinnata (Huds.) Papenf. \\ GIGARTINALES \\ Cruoriaceae \\ CRUORIA Fries, I835. \\ pellita (Lyngb.) Fries}

GELIDIUM Lamouroux, I8I3. (8) (capillacea (Gmel.) Born. et Thur.) rosea Crn.

var. rosea

var. purpurea (Crn.) Batt.

CRUORIOPSIS Dufour, I864. gracilis (Kuck.) Batt. ? hauckii Batt. (9)

Petrocelis J. Agardh, I852. cruenta J. Ag. hennedyi (Harv.) Batt.

Calosiphoniaceae

CALOSIPHONIA Crouan, I852. vermicularis (J. Ag.) Schm.

Nemastomaceae (10)

Platoma Schmitz, I889. bairdii (Farl.) Kuck. marginifera J. Ag.

SCHIZYMENIA J. Agardh, I85I. dubyi (Chauv.) J. Ag.

\section{Furcellariaceae}

FURCELlARIA Lamouroux, I8I3. fastigiata (L.) Lamour. (11)

HALARACHNION Kützing, I843. ligulatum (Woodw.) Kütz.

\section{Rhabdoniaceae}

Catenella Greville, I83o. repens (Lightf.) Batt.

Rhodophyllidaceae

CALLIBLEPHARIS Kützing, I843. ciliata (Huds.) Kütz. var. ciliata var. ? angusta Holm. et Batt. lanceolata (Stackh.) Batt.

CystoclonIUM Kützing, I843. purpureum (Huds.) Batt.

RHODOPHYLLIS Kützing, I847. ? appendiculata J. Ag. divaricata (Stackh.) Papenf. (12) (bifida Kütz.)

\section{Plocamiaceae}

Plocamium Lamouroux, I8I3. coccineum (Huds.) Lyngb.
f. coccineum
f. uncinatum (Ag.) Levring

Sphaerococcaceae

SPHAEROCOCCUS Stackhouse, I797. coronopifolius Stackh.

\section{Gracilariaceae}

CoRDYlecladia J. Agardh, 1852. erecta (Grev.) J. Ag.

GRACILARIA Greville, I83o. bursa-pastoris (Gmel.) Silva (compressa (Ag.) Grev.) foliifera (Forssk.) Børg. (multipartita J. Ag.) verrucosa (Huds.) Papenf. (confervoides (L.) Grev.)

f. verrucosa

f. gracilis (Stackh.)

f. procerrima (Turn.) 
Phyllophoraceae

AHNFELTIA Fries, I835. plicata (Huds.) Fries

GYMNOGONGRUS Martius, I833. griffithsiae (Turn.) Mart. norvegicus (Gunn.) J. Ag. patens J. Ag.

? Petroglossum Hollenberg, - 1943. nicaeense (Duby) Schotter (13)

(Rhodymenia palmetta var. elisiae Chauv.)

Phyllophora Greville, I830. (14) brodiaei (Turn.) J. Ag. membranifolia (Good. et Woodw.) J. Ag. palmettoides J. Ag. rubens (L.) Grev. (epiphylla (Müll.) Batt.) traillii Holm. et Batt.

STENOGRAmme Harvey, I84I. interrupta (Ag.) Mont.

\section{Gigartinaceae}

CHONDRUS Stackhouse, I797. crispus (L.) Stackh. (15)

GigarTINA Stackhouse, I809. acicularis (Wulf.) Lamour. pistillata (Gmel.) Stackh. stellata (Stackh.) Batt. (15) teedii Lamour.

\section{CRYPTONEMIALES}

Gloiosiphoniaceae

Gloiosiphonia Carmichael ex Berkeley, I833. capillaris (Huds.) Carm.

\section{Dumontiaceae}

DILSEA Stackhouse, I809. carnosa (Schmidel) Kuntze (edulis (Stackh.) Stackh.)

DUDRESNAYA Crouan frat., I835. verticillata (With.) Le Jol.

DumONTIA Lamouroux, I8I3. incrassata (Müll.) Lamour.

\section{Polyideaceae}

POLYIDES Agardh, I822. caprinus (Gunn.) Papenf. (rotundus (Gmel.) Grev.)

Squamariaceae (16)

ERYTHRODERMIS Batters, I900. (17) allenii Batt.

HAEMATOCELIS J. Agardh, I852. (17) rubens J. Ag.

PeysSONELIA Decaisne, I84I. atropurpurea $\mathrm{Crn}$. dubyi Crn. (Cruoriella dubyi (Crn.) Schm.) harveyana Crn. rosenvingii $\mathrm{Schm}$. rubra (Grev.) J. Ag. rupestris Crn.

PoRPHYRODISCUS Batters, I897. (17) simulans Batt.
RHODODERMIS Crouan, I852. elegans Crn. var. elegans var. polystromatica Batt. (18) georgii (Batt.) Collins (Rhodophysema georgii Batt.) ? parasitica Batt. (19)

RHoDodiscus Crouan, I859. pulcherrimus Crn.

Hildenbrandiaceae

HildENBRANDIA Nardo, I834. crouanii J. Ag. prototypus Nardo

Corallinaceae (20)

Choreonema Schmitz, I889. thuretii (Born.) Schm.

CORALlina Linnaeus emend. Lamouroux, I812. ? elongata Johnst. (21) granifera Ellis et Soland. (virgata Zanard.)

officinalis L.

f. officinalis

f. compacta (Crn.) squamata Ellis

EPILITHON Heydrich, I897. (22) ? membranaceum (Esper) Heydr. (Lithothamnion membranaceum Fosl.)

JANIA Lamouroux, I8I2. corniculata (L.) Lamour.

(Corallina rubens var. corniculata Hauck) rubens (L.) Lamour.

(Corallina rubens Ellis et Sol.)

LITHOPHYLlUM Philippi, I837. adplicitum (Fosl.) Newton corallinae (Crn.) Heydr.

(Melobesia corallinae Crn.) crouanii Fosl.

fasciculatum Fosl.

f. complanata Fosl

f. divergens Fosl.

f. incrassata Fosl. hapalidioides (Crn.) Heydr.

f. hapalidioides

f. confinis (Crn.) Fosl.

incrustans Phil.

f. incrustans

f. depressa (Crn.) Fosl.

f. harveyi Fosl.

f. subdichotomum Heydr. orbiculatum Fosl. (23) pustulatum (Lamour.) Fos1.

f. pustulatum

f. laminariae (Crn.) Fosl.

LithothamnIon Philippi, I837. bornetii Fosl. calcareum (Pall.) Aresch.

f. calcareum

f. compressa (McCalla) Fosl.

f. crassa Lem.

f. squarrulosa Fosl. 
LithothamNION (cont.)

calcareum (cont.)

f. subsimplex (Batt.) Fosl.

f. ? subvalida Fosl.

? colliculosum Fosl. (24)

f. colliculosum

f. rosea Batt.

compactum Kjellm.

? fruticulosum (Kütz.) Fosl.

glaciale Kjellm.

granii Fosl. (24)

f. reducta Fosl. (battersii Fosl.)

laeve (Strömf.) Fosl.

(stroemfeltii Fosl.)

laevigatum Fosl.

lenormandii (Aresch.) Fosl.

f. lenormandii

f. squamulosa Fosl.

f. sublaevis Fosl

norvegicum (Aresch.) Kjellm.

(calcareum var. norvegicum Fosl.)

polymorphum (L.) Aresch.

sonderi Hauck

f. sonderi

f. sublaevigata Fosl.

MELobesia Lamouroux, I8I2. (22)

? farinosa Lamour.

var. farinosa

var. borealis Lemoine

var. solmsiana Fkbg.

? lejolisii Rosan.

? minutula Fosl.

? zonalis (Crn.) Fosl.

Mesophyllum Lemoine, I928.

lichenoides (Ellis) Lemoine

(Lithothamnion lichenoides Fosl.)

f. lichenoides

f. agariciformis (Johnst. ?)

f. depressa (Fosl.)

SCHMitzIELla Bornet et Batters, I892. endophlaea Born. et Batt.

\section{Grateloupiaceae}

GRATELOUPIA Agardh, I822.

dichotoma J. Ag.

filicina (Wulf.) Ag.

var. filicina

var. intermedia Holm. et Batt. minima Crn.

HALYMENIA Agardh, I8I7. latifolia Crn.

Kallymeniaceae

Callocolax Schmitz, I895. neglectus Schm.

CALLOPHYLlis Kützing, I843.

flabellata Crn.

laciniata (Huds.) Kütz.

EUTHORA J. Agardh, I847. cristata (L.) J. Ag.

KALLYMENIA J. Agardh, I842. (25)

? larterae Holm.

reniformis (Turn.) J. Ag.

var. reniformis

var. ferrarii J. Ag.

var. undulata J. Ag.
Meredithia J. Agardh, I892. microphylla J. Ag.

(Kallymenia microphylla J. Ag.)

Choreocolaceae

CHOREOCOLAX Reinsch, I875. polysiphoniae Reinsch

HARVEYella Schmitz et Reinke, I889. mirabilis (Reinsch) Schm. et Rke.

Holmsella Sturch, I926. pachyderma (Reinsch) Sturch

BONNEMAISONIALES (26)

Naccariaceae

ATRACTOPHORA Crouan, I849. hypnoides $\mathrm{Crn}$.

NACCARIA Endlicher, I836. wiggii (Turn.) Endl.

\section{Bonnemaisoniaceae}

AsPARAGOPSIS Montagne, I840. armata Harv.

tetrasporophyte $=$ Falkenbergia rufolanosa (Harv.) Schm.

BonNemaisonia Agardh, I822. asparagoides (Woodw.) Ag. tetrasporophyte $=$ Hymenoclonium serpens (Crn.) Batt.

clavata (Schousb.) Hamel

hamifera Hariot tetrasporophyte $=$ Trailliella intricata Batt.

\section{RHODYMENIALES}

Champiaceae

CHAMPIA Desvaux, I808.

parvula (Ag.) Harv.

Chylocladia Greville, I833.

reflexa Lenorm.

squarrosa (Kütz.) Le Jol. (kaliformis var. squarrosa Harv.)

verticillata (Lightf.) Bliding

(kaliformis(Good.et Woodw.) Grev.) var. verticillata

var. patens (Kütz.)

GASTROCLONIUM Kützing, I843.

ovatum (Huds.) Papenf.

(Chylocladia ovata Batt.)

var. ovatum

var. subarticulatum (Kütz.)

LOMENTARIA Lyngbye, I8I9.

articulata (Huds.) Lyngb.

clavellosa (Turn.) Gaill.

var. clavellosa

var. sedifolia Harv.

orcadensis (Harv.) Collins (rosea (Harv.) Thur.)

Rhodymeniaceae

RHODYMENIA Greville, I830.

ardissonei J. Feldm. (corallicola Ardiss.)

palmata (L.) Grev. (27)

pseudopalmata (Lamour.) Silva (27) 


\section{CERAMIALES}

\section{Ceramiaceae}

Aglaothamnion G. FeldmannMazoyer, I940.

brodiaei (Harv.) G. Feldm.

(Callithamnion brodiaei Harv.)

polyspermum (Ag.) Parke

(Callithamnion polyspermum Ag.)

f. polyspermum

f. scopulorum (Ag.) Parke

(Aglaothamnion scopulorum

(Ag.) G. Feldm.

tenuissimum (Bonnem.) G. Feldm.

(Callithamnion tenuissimum

(Bonnem.) Kütz.)

tripinnatum (Grat.) G. Feldm.

(Callithamnion tripinnatum

(Grat.) Ag.)

AntithamnIoN Nägeli, I847.

boreale (Gobi) Kjellm.

cruciatum (Ag.) Näg.

var. cruciatum

var. pumilum (Harv.) Batt.

floccosum (Müll.) Kleen

plumula (Ellis) Thur.

var. plumula

var. crispum (Ducluz.) Hauck

var. spinescens Strömf.

sarniense (Lyle) G. Feldm. (28)

(Antithamnionella sarniensis Lyle) spirographidis Schiffner

BORNETIA Thuret, I855. secundiflora (J. Ag.) Thur.

Callithamnion Lyngbye emend.

G.Feldmann-Mazoyer, I940. (29) arbuscula (Dillw.) Lyngb.

byssoides Arnott

corymbosum (Sm.) Lyngb.

dudresnayi Crn.

? fruticulosum J. Ag. (30)

granulatum (Ducluz.) Ag. (31)

hookeri (Dillw.) Ag.

rabenhorstii Crn.

? roseum Harv. (32)

? spongiosum Harv. (31)

tetragonum (Wither.) Ag. (30)

f. tetragonum

f. brachiatum (Bonnem.) K. Rosenv. tetricum Ag.

Ceramium Roth, I797. (33)

acanthonotum Carm. ex Harv.

arborescens $\mathrm{J}$. Ag.

areschougii Kylin

atlanticum Peterson

boergesenii Peterson

ciliatum (Ellis) Ducluz.

circinatum (Kütz.) J. Ag.

? crouanianum J. Ag.

derbesii Solier

deslongchampsii Chauv.

diaphanum (Lightf.) Roth

echionotum J. Ag.

var. echionotum

var. transcurrens (Kütz.) Batt. fastigiatum Harv.

flabelligerum J. Ag.

fruticulosum (Kütz.) J. Ag. gracillimum Griff. et Harv. pedicellatum (Duby) J. Ag. pennatum Crn.

rubrum (Huds.) Ag.

secundatum J. Ag.

strictum Harv.

tenue J. Ag.

tenuissimum (Lyngb.) J. Ag.

vimineum J. Ag.

Compsothamnion Nägeli, I86r. gracillimum (Harv.) Näg.

thuyoides (Sm.) Näg.

CORYNOSPORA J. Agardh emend. J. Agardh, I876.

pedicellata (Sm.) J. Ag.

(Monospora pedicellata (Sm.) Solier)

f. pedicellata

f. comosa (Holm. et Batt.)

Crouninia J. Agardh, I842. attenuata (Bonnem.) J. Ag.

GRIFFITHSIA Agardh, I8I7. barbata (Sm.) Ag. corallinoides (L.) Batt.

devoniensis Harv. (34) flosculosa (Ellis) Batt.

HALURUS Kützing, I849. equisetifolius (Ag.) Kütz. var. equisetifolius var. simplicifilum $\mathrm{J}$. Ag.

Microcladia Greville, I83o. glandulosa (Soland.) Grev.

PleONOSPORIUM Nägeli emend. Nägeli ex Hauck, I883.

borreri (Sm.) Näg. ex Hauck var. borreri

var. fasciculatum (Harv.) Holm. et Batt.

? Plumaria Schmitz, I896. (35)

? elegans (Bonnem.) Schm.

? PTILOTa Agardh, I8I7. (35)

? plumosa (Huds.) Ag.

Ptilothamnion Thuret, I863.

? lucifugum Cotton (36)

pluma (Dillw.) Thur.

SEIROSPORA Harvey, I846. (37)

griffithsiana Harv.

hormocarpa (Holm.) Batt.

interrupta (Sm.) Schm.

SPERMOTHAMNION Areschoug, I877. (38) barbatum (Ag.) Näg.

irregulare (J. Ag.) Ardiss.

repens (Dillw.) K. Rosenv.

(roseolum Pringsh.) (turneri (Mert.) Aresch.) strictum (Ag.) Ardiss.

SPHONDYLOTHAMNION Nägeli, I86r. multifidum (Huds.) Näg. var. multifidum var. piliferum (Ag.) Batt.

SPyridia Harvey, I833.

filamentosa (Wulf.) Harv. 


\section{Delesseriaceae}

ACrosorium Zanardini, I869. reptans (Crn.) Kylin (39) uncinatum (J. Ag.) Kylin (39)

Apoglossum J. Agardh, I898. ruscifolium (Turn.) J. Ag.

CRYPTOPLEURA Kützing, I843. ramosa (Huds.) Kylin
f. ramosa
f. ciliifera (Kütz.)
f. lobata (Kütz.)
f. uncinata (Grev.)

DELESSERIA Lamouroux, I8I3. sanguinea (Huds.) Lamour.

ERYTHROGLOSSUM J. Agardh, I898. sandrianum (Zanard.) Kylin

GoNIMOPHYLLUM Batters, I892. buffhamii Batt.

HYPOGLOSSUM Kützing, I843. woodwardii Kütz. (40)

Membranoptera Stackhouse, I809. alata (Huds.) Stackh.

Nitophyllum Greville, I830. (41)

? bonnemaisonii Grev.

(Myriogramme bonnemaisonii Kylin)

var. bonnemaisonii

var. crassinerve Batt. punctatum (Stackh.) Grev.

? versicolor Harv.

(Myriogramme versicolor Kylin)

PANTONEURA Kylin, I9I9. angustissima (Turn.) Kylin

PHYCODRYs Kützing, I843. rubens (Huds.) Batt.

POLYNEURA Kylin, I924. gmelinii (Grev.) Kylin hilliae (Grev.) Kylin litterata (J. Ag.) Kylin

RHIzOGLOSSUM Kylin, I924. thysanorhizans (Holm.) Kylin

\section{Dasyaceae}

DASYA Agardh, I824. arbuscula (Dillw.) Ag.

var. arbuscula

var. ? caespitosa J. Ag.

corymbifera J. Ag.

ocellata (Grat.) Harv. punicea Menegh.

HETEROSIPHONIA Montagne, I842. plumosa (Ellis) Batt.

$$
\begin{aligned}
& \text { f. plumosa } \\
& \text { f. patens (Grev.) } \\
& \text { f. tenuior (Dillw.) }
\end{aligned}
$$

\section{Rhodomelaceae (42)}

BostrychIA Montagne, I8 38 . scorpioides (Huds.) Mont.

BRONGNIARTELLA Bory, I822. byssoides (Good. et Woodw.) Schm.

CHONDRIA Agardh, I8I7. caerulescens J. Ag.

dasyphylla (Woodw.) Ag. tenuissima (Good. et Woodw.) Ag.
HaLOPITYS Kützing, I843. incurvus (Huds.) Batt.

LAURENCIA Lamouroux, I8I3. (43) hybrida (D.C.) Lenorm. ex Duby (caespitosa Lamour.) obtusa (Huds.) Lamour. pinnatifida (Huds.) Lamour.

LOPHOSIPHONIA Falkenberg, I897. obscura (Ag.) Fkbg.

(Polysiphonia obscura J. Ag.)

ODONTHALIA Lyngbye, I8I9. dentata (L.) Lyngb.

PolysirHonIA Greville, I824. (44) brodiaei (Dillw.) Grev. denudata (Dillw.) Kütz. (variegata (Ag.) Zanard.) elongata (Huds.) Harv. elongella Harv.

fibrata (Dillw.) Harv.

fibrillosa (Dillw.) Grev. (45)

foetidissima Cocks

fruticulosa (Wulf.) Spreng.

(Pterosiphonia fruticulosa (Wulf.) Batten)

furcellata (Ag.) Harv.

var. furcellata

var. forcipata J. Ag.

insidiosa Crn.

lanosa (L.) Tandy (fastigiata Grev.)

macrocarpa Harv.

nigra (Huds.) Batt.

nigrescens (Sm.) Grev.

f. nigrescens

f. affinis (Moore) J. Ag.

f. fucoides (Huds.) J. Ag.

f. protensa J. Ag.

f. senticosa J. Ag.

opaca (Ag.) Zanard.

rhunensis Thur.

richardsonii Hook.

? sanguinea (Ag.) Zanard.

simulans Harv.

spinulosa Grev.

var. spinulosa

var. major J. Ag.

spiralis Batten

subulifera (Ag.) Harv.

var. subulifera

var. templetonii Harv.

urceolata (Dillw.) Grev.

f. urceolata

f. comosa (Ag.) J. Ag.

f. formosa (Suhr) J. Ag.

f. patens (Dillw.) J. Ag. violacea (Roth) Grev. (46)

PTEROSIPHONIA Falkenberg, I889. complanata (Clem.) Fkbg. parasitica (Huds.) Fkbg. pennata (Roth) Fkbg. thuyoides (Harv.) Schm.

RHODOMELA Agardh, I822. (47) confervoides (Huds.) Silva (subfusca (Woodw.) Ag.) lycopodioides (L.) Ag. 
(1) See Waern (1952).

\section{Notes on Rhodophyceae}

(2) See Hamel (1924, p. 283).

(3) British material of genus in need of revision.

(4) According to Koster (1952) should be B. atropurpurea (Roth) Ag. f. fuscopurpurea (Dillw.) Ag. as there are no differences to be found between the fresh-water and marine material; see also Hamel (1924), and Kylin (1944). Kept the marine form distinct for the present awaiting the results of Dr K. M. Baker's investigations.

(5) See Drew (1949).

(6) Acrochaetium Nägeli, sens. lat.

(7) Dr K. M. Baker is revising the British material of this genus.

(8) Awaiting the results of the investigation of this genus carried out by Dr M. de Valèra and Mr P. Dixon.

(9) Requires investigation.

(10) Nemastoma J. Agardh, I842; N. dichotoma J. Ag., recorded by Lyle (I920) for Guernsey. J. Feldmann (I94I) thinks that several species may have been confused under the above name, so Lyle's specimen should be examined.

(11) See Papenfuss (1950), Waern (1952), Silva (1952).

(12) Rhodophyllis bifida var. incrassata Harv., requires investigation before any new combination is made under $R$. divaricata. $R$. appendiculata may be a form of $R$. divaricata (J. Feldmann, I94I).

(13) See J. Feldmann (I94I, p. 86) and G. Schotter (1952).

(14) The form-range of the species on the British coast require investigation.

(15) See Marshall, Newton \& Orr (1949).

(16) British material belonging to this family requires monographic treatment.

(17) Systematic position uncertain.

(18) See Cotton (I9I2).

(19) See Kylin (1944).

(20) In need of monographic treatment.

(21) Needs investigation-should be possibly C. mediterranea Aresch. or C. officinalis L. var. mediterranea (Aresch.) Hauck.

(22) The genus Fosliella Howe not adopted for the present-appears to require further investigation. If adopted the British species would be $F$. farinosa (Lamour.) Howe, F. lejolisii (Rosan.) Howe, F. minuta (Fosl.), F. zonalis (Crn.) J. Feldm., and Epilithon membranaceum would again be known as Melobesia membranacea (Esper.) Lamour.

(23) Should possibly be placed in genus Pseudolithothamnion Lemoine, I913; see Lemoine (1928).

(24) Regarded by some workers as a form of L. glaciale.

(25) $K$. larterae Holm. requires investigation-may be a form of $K$. reniformis (Turn.) J. Ag.

(26) See J. \& G. Feldmann (1942, I952) and J. Feldmann (1952).

(27) The form-range of the species requires investigation and revision.

(28) Possibly a form of $A$. spirographidis, see J. Feldmann (1942).

(29) British species require investigation as no doubt some of the species listed here under Callithamnion should be placed under Aglaothamnion. The range of form of species of this genus on the British coast is in urgent need of attention.

(30) Kolderup Rosenvinge (1924) and Kylin (1944) consider C. tetragonum, C. spiniferum Kylin (Dutch coast) and C. fruticulosum forms of one species. Kolderup Rosenvinge (1924) unites them under C. tetragonum but Kylin (1944) prefers to keep them separate and to use the name C. tetragonum for plants on the British coast and C. fruticulosum for the plants on the Swedish coast. The form-range of the series on the British coast needs investigation and comparison with material from Sweden, Holland and Denmark.

(31) Cotton (I912) thinks that C. spongiosum and C. granulatum may be two distinct species, having found no intermediate forms between them. They have been listed separately awaiting monographic treatment of British material.

(32) Left as C. roseum Harv. until it is certain that Harvey's plant is the same as C. roseum (Roth) Lyngb. 
(33) British material in urgent need of revision, see work of Petersen, Kylin, Sjösted), Waern (references to literature in Waern, I952), Kolderup Rosenvinge (1924), G. Feldmann-Mazoyer (1940), Lucas (1950). The different workers' interpretations of many of the species and of the form-range within any one species vary so much that no attempt has been made to link up the 'species' recorded for the British coast with these treatments. This must await monographic treatment of British material.

(34) Possibly an ecological form of G. corallinoides, Koster (I952).

(35) Plumaria Schm. and Ptilota Ag. used awaiting decision concerning conservation of Plumaria Schm. over Plumaria Stackh.-see Silva (1952).

(36) See Cotton (I9I2), G. Feldmann-Mazoyer (I940); P. lucifugum Cotton may be a form of $P$. pluma since G. Feldmann considers that $P$. micropterum (Mont.) Born. is a reduced form of $P$. pluma. Fertile material of Cotton's plant needs investigation.

(37) British material in need of revision.

(38) British material requires investigation and revision. Kolderup Rosenvinge places S. turneri (Mert.) Aresch. as var. turneri (Mert.) K. Rosenv. and S. roseolum (Ag.) Pringsh. as var. roseolum (Ag.) K. Rosenv. under S. repens (Dillw.) K. Rosenv. If Conferva repens Dillw. is the same plant as that described by Mertens as Ceramium turneri, which Kolderup Rosenvinge believed it was, then var. turneri should be placed as a synonym under S. repens (Dillw.) K. Rosenv. var. repens. See J. Feldmann (1942, p. 59) concerning $S$. strictum.

(39) Doubtful if these two species are distinct.

(40) Forms of this species occurring on British coast in need of revision.

(41) Kylin (1934) considered that N. bonnemaisonii and N. versicolor are closer to the genus Nitophyllum than to the genus Myriogramme Kylin and so should remain in the genus Nitophyllum for the present; he considered, however, that they are sufficiently different from the type species $N$. punctatum to warrant their being placed in a new genus.

(42) Ctenosiphonia Falkenberg, I897; C. hypnoides (Welw.) Fkbg. recorded for Guernsey by Lyle (1920, I923).

(43) Form-range of species on British coast needs revision.

(44) Form-range of species on British coast requires investigation.

(45) Considered by some workers a form of $P$. violacea; British material needs investigation.

(46) The forms of this species on the British coast need investigation, see Kolderup Rosenvinge (1924), Kylin (I944), Veldkamp (1950). P. subulata (Ducluz.) J. Ag. considered a distinct species by some workers and not a variety of $P$. violacea.

(47) Forms of $R$. confervoides require revision before any new combinations are made. $R$. lycopodioides should possibly be included as a variety under $R$. confervoides.

\section{CYANOPHYCEAE (1)}

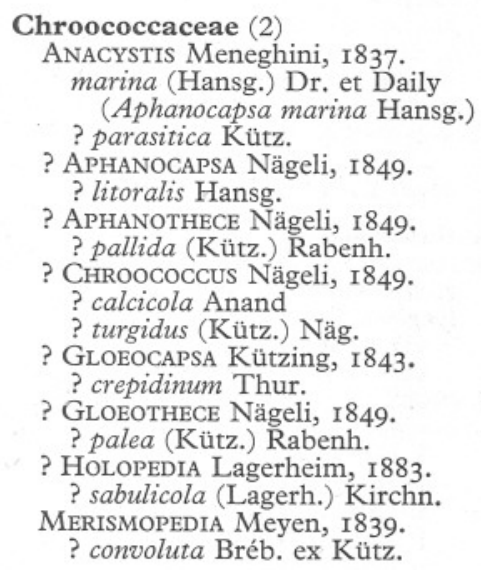

? glauca (Ehrb.) Näg.
f. glauca
f. ? mediterranea (Näg.) Collins
? revoluta Ask.

? SYNECHOCOCCUS Nägeli, I849.

? bacillaris Butcher

Chamaesiphonaceae $(2,3)$

? Chamaesiphon A. Braun. et Grunow, $\mathrm{I} 865$.

? marinus Wille (4)

? Chlorogloea Wille, I900.

? tuberculosa (Hansg.) Wille

? Dermocarpa Crouan, I858.

? enteromorphae Anand

? leibleiniae (Reinsch) Born. et Thur.

? olivacea (Reinsch) Tilden

? incrustans (Reinsch) Batt.

? rosea (Reinsch) Batt.

? sphaerica Setch. et Gardn. ex Gardn. 
? DERMOCARPA (cont.)

? violacea Crn.

ENTOPHYSALIS Kützing, I843. conferta (Kütz.) Dr. et Daily (Dermocarpa prasina (Reinsch) Born. et Thur.) (Xenococcus schousboei Thur.) crustacea (Ag.) Dr. et Daily (granulosa Kütz.) (Hyella caespitosa Born. et Flah.) (Pleurocapsa fuliginosa Hauck)

? ONCOBYRSA Agardh, I827.

? marina (Grun.) Rabenh.

? Pleurocapsa Thuret, I885. ? amethystea $\mathrm{K}$. Rosenv.

? crepidinum Collins

? entophysaloides Setch. et Gardn. ex Gardn.

? kerneri (Hansg.) Dr.

? Xenococcus Thuret ex Bornet et Thuret, r880.

? gilkeyae Setch. et Gardn. ex Gardn. (forma)

? pyriformis Setch. et Gardn. ex Gardn.

? violacea Anand

\section{Stigonemataceae}

BRACHYTRICHIA Zanardini; Bornet et Flahault, I886.

balani (Lloyd) B. et F.

MASTIgocoleus Lagerheim; Bornet et Flahault, I887.

testarum Lagerh. ex. B. et F.

\section{Nostocaceae}

ANABAENA Bory; Bornet et Flahault, I888.

? berkeleyana (Thwaites) Batt.

? broomei (Thwaites) Batt.

torulosa (Carm.) Lagerh. ex B. et F. variabilis Kütz. ex $\mathrm{B}$. et $\mathrm{F}$.

Nodularia Mertens; Bornet et Flahault, I888.

harveyana (Thwaites) Thur. ex. B. et $\mathrm{F}$.

spumigena Mert. ex. B. et F. var. litorea (Kütz.) B. et F.

Nostoc Vaucher; Bornet et Flahault, I 888 .

commune Vauch. ex B. et F.

entophytum B. et F.

linckia (Roth) Born. ex B. et F.

Pseudanabaena Lauterborn, i9i6. brevis Carter

\section{Rivulariaceae}

AMPHITHRIX Kützing; Bornet et Flahault, I886.

violacea (Kütz.) B. et $\mathrm{F}$.

CALOTHRIX Agardh; Bornet et Flahault, I886.

aeruginea (Kütz.) Thur. ex B. et F. confervicola $\mathrm{Ag}$. ex B. et $\mathrm{F}$.

var. confervicola

var. purpurea $\mathrm{B}$. et $\mathrm{F}$.
? consociata (Kütz.) B. et F. (5)

? contarenii B. et F. (6)

cottonii nov. nom. (7)

(endophytica Cotton)

crustacea Thur. ex B. et F.

fusca (Kütz.) B. et F.

litoralis Anand

parasitica (Chauv.) Thur. ex. B. et F.

pulvinata (Ag.) B. et F.

var. pulvinata

var. prostrata Anand

scopulorum (Web. et Mohr) Ag.

ex B. et F.

(fasciculata Ag. ex B. et F.) (8)

vivipara Harv. ex $\mathrm{B}$. et $\mathrm{F}$.

Dichothrix Zanardini; Bornet et Flahault, I886.

gypsophila (Kütz.) B. et F.

IsACTIS Thuret; Bornet et Flahault, I886.

plana Thur. ex B. et F.

var. plana

var. fissurata B. et F.

RIVULARIA Roth; Bornet et Flahault, I886.

atra Roth ex B. et F. (9)

australis Harv. ex B. et F.

biasolettiana Menegh. ex B. et F.

bullata (Poir.) Berk. ex B. et F.

mesenterica Thur. ex B. et F.

nitida Ag. ex B. et F. (9)

\section{Scytonemataceae}

? Diplocolon Nägeli; Bornet et Flahault, I887. (10)

? codii Batt. (10)

Fremyella J. De Toni, I936. (MICROCHAETE Thuret, I875) aeruginea (Batt.) nov.comb. (Microchaete aeruginea Batt.) grisea (Thur. ex B. et F.) J. De Toni (Microchaete grisea Thur.)

Plectonema Thuret; Gomont, I893.

battersii Gom.

intorale Anand

norvegicum Gom.

nostocorum Born. ex Gom.

terebrans Born. et Flah. ex Gom.

TOLYPOTHRIX Kützing; Bornet et Flahault, I887.

tenuis Kütz. ex B. et $\mathrm{F}$.

\section{Oscillatoriaceae}

HYDROCOLEUM Kützing; Gomont, 1892. glutinosum (Ag.) Gom.

lyngbyaceum Kütz. ex Gom.

var. lyngbyaceum

var. rupestre Kütz. ex Gom.

LyNGBYA Agardh; Gomont, I893. aestuarii Liebm. ex Gom. (11)

f. aestuarii

f. aeruginosa Gom.

f. ferruginea Gom.

f. limicola Gom.

f. natans Gom. 
LYNGBYA (cont.)

aestuarii (cont.)

f. spectabilis Gom.

f. symplocoidea Gom. agardhii Gom.

confervoides Ag. ex. Gom. gracilis Rabenh. ex Gom.

var. maritima Anand

lutea Gom. ex Gom.

majuscula Harv. ex Gom.

meneghiniana Gom. ex Gom.

rivulariarum Gom.

semiplena J. Ag. ex Gom.

Microcoleus Desmazières; Gomont, I892.

acutirostris Gom.

chthonoplastes Thur. ex Gom.

tenerrimus Gom.

OsCILlATORIA Vaucher; Gomont, I893. (12)

amphibia Ag. ex Gom.

bonnemaisonii Crn. ex Gom.

brevis Kütz. ex Gom.

var. neapolitana Gom.

corallinae Gom. ex Gom.

formosa Bory ex Gom.

laetevirens Crn. ex Gom.

limosa Ag. ex Gom.

margaritifera Kütz. ex Gom.

nigro-viridis Thwaites ex Gom. sancta Kütz. ex Gom.

PhORMIdIUM Kützing; Gomont, I893. ambiguum Gom.

angustissimum W. et G. S. West autumnale Gom.

corium Gom. ectocarpi Gom.

foveolarum Gom.

f. foveolarum

f. minus Anand

f. moniliforme Anand

fragile Gom.

monile Setch. et Gardn. (forma)

papyraceum Gom. ex Gom.

persicinum Gom.

submembranaceum Gom.

var. minor Anand

subuliforme Gom.

tenue Gom.

uncinatum Gom. ex Gom.

valderianum Gom.

SCHIZOTHRIx Kützing; Gomont, I892. cresswellii Harv. ex Gom.

fritschii Anand

lardacea Gom. vaginata Gom.

SPIRULINA Turpin; Gomont, I893. major Kütz. ex Gom.

subsalsa Oerst. ex Gom.

f. subsalsa

f. oceanica Gom.

subtilissima Kütz. ex Gom. versicolor Cohn ex Gom. (13)

SyMPLOCA Kützing; Gomont, I893. atlantica Gom.

var. atlantica

var. purpurea Batt.

dubia Gom.

hydnoides Kütz. ex Gom.

var. hydnoides

var. fasciculata Gom.

\section{Notes on Cyanophyceae}

(1) For various systems of classification see Geitler (1932), Frémy (1934), Fritsch (1945), Smith (I950); families only given in list.

(2) See Drouet (I95I) and Drouet \& Daily (1952) who are also preparing for publication a detailed revision of the coccoid Cyanophyceae. Dr F. Drouet has very kindly given the following information for inclusion here. In the revision of the Chroococcaceae the genus Anacystis Menegh. will include Aphanocapsa, Chroococcus and Gloeocapsa of this list and the genus Coccochloris Spreng. will include Aphanothece, Gloeothece, and Synechococcus of this list. In the revison of the Chamaesiphonaceae the genus Entophysalis Kütz. will include all the genera given under this family in this list including Pleurocapsa Thur. as to type, but not as to many of the species which largely are forms of Anacystis.

(3) See also Nadson (1932).

(4) Probably not a member of the Cyanophyceae.

(5) $=C$. confervoides Ag.?

(6) Possibly a form of C. scopulorum Ag.

(7) = Calothrix endophytica Cotton, Proc. Roy. Irish Acad., Vol. 3r, p. I04, I9I2 [non Calothrix endophytica Lemmerman, Forsch. Ber. d. Biolog. Stat. zu Plön, p. I84, I896 = Homoeothrix endophytica (Lemm.) Lemm., Krypt. Flor. Mark Brandenburg, Bd. 3, p. 240, I910.]

(8) Both Lindstedt (I943) and Du Rietz (I947) consider that C. fasciculata Ag. is a luxuriant growth form of C. scopulorum. The plant figured and described as C. fasciculata by Harvey in Phyc. Brit., may be, according to some authorities, a species quite distinct from the true C. fasciculata $\mathrm{Ag}$.

(9) Geitler (1932) suggests that R. atra is possibly the young stage of $R$. nitida. 
(10) The value of this genus appears to be debatable since $D$. heppii Näg., on which the genus was founded, is, according to some authorities, only a form of Scytonema crustaceum Ag. There also seems to be doubt concerning Batters' plant (Frémy, I934) since his diagrams do not show false branching but true branching-this requires investigation.

(11) Intermediates occur between all the described forms (Frémy, I934).

(12) Two species omitted from list as they are in need of investigation. (a) O. rosea (Crn.) Batt. [non O. rosea Utermöhl, 1925, =O. utermoehlii J. De Toni, I939] given as a sp. inquir. in Gomont. (b) O. subuliformis Thwaites ex Harv., Phyc. Brit.; does Thwaites' plant $=O$. subuliformis Kütz.?

(13) Probably a form of $S$. subsalsa-no essential difference between the two species (Geitler, I932).

\section{REFERENCES}

Batters, E. A. L., 1906. New or critical British marine Algae. Fourn. Bot., Lond., Vol. 44, pp. I-3.

BlIdING, C., I944. Zur Systematik des schwedischen Enteromorphen. Bot. Notiser, I944, pp. 33I-56.

Chadefaud, M., I94I. Sur l'organisation et la position systématique des Flagellés du genre Pyramidomonas. Rev. Sci., Paris, 79 année, pp. II3-I4.

— 1945. Sur la position systématique des Vauchéries. Bull. Soc. bot. Fr., T. 92, pp. $47-50$.

— 195I. Les Vauchéries sont des Xanthophycées. Bull. Soc. bot. Fr., T. 98, pp. 2 IO-II.

Chapman, V. J., I939. Some algal complexities. Rhodora, Vol. 4I, pp. 19-28.

- 1952. New entities in the Chlorophyceae of New Zealand. Trans. Roy. Soc. N.Z., Vol. 80, pp. 47-58.

Christensen, T., I952. Studies on the genus Vaucheria. I. A list of finds from Denmark and England with notes on some submarine species. Bot. Tidsskr., Bd. 49 , pp. I7I-88.

Cotton, A. D., I9I2. Clare Island Survey. Pt. I5. Marine Algae. Proc. Roy. Irish Acad., Vol. 31, I78 pp.

Drew, K. M., I949. Conchocelis-phase in the life-history of Porphyra umbilicalis (L.) Kütz. Nature, Lond., Vol. I64, p. 748.

Drouet, F., I95I. Cyanophyta. Chapter 8 in Manual of Phycology. Ed. G. M. Smith. Waltham, Mass.

Droute, F. \& DaILy, W. A., I952. A synopsis of the coccoid Myxophyceae. Butler Univ. Bot. Stud., Vol. Io, pp. 220-23.

Du RIETZ, G. E., I940. On the identity of Dictyosiphon chordaria Areschoug and Gobia baltica (Gobi) Reinke. Svensk bot. Tidskr., Bd. 34, pp. 35-46.

- 1947. Wellengrenzen als ökologische Äquivalente der Wasserstandslinien. Zool. Bidr. Uppsala, Bd. 25, pp. 534-50.

FeldmanN, J., I94I. Les Algues Marines de la Côte des Albères. IV. Rhodophycées. Fasc. II (Gigartinales, Rhodymeniales). Rev. Algol., T. I2, pp. 77-100.

- 1942. Les Algues Marines de la Côte des Albères. IV. Rhodophycées. Fasc. III (Céramiales). Trav. Algol., Vol. I, pp. 29-II3.

— 1949. L'ordre des Scytosiphonales. Mém. Soc. Hist. Nat. Afr. N., Hors-série, T. 2, pp. $103-15$.

- I952. Les cycles de reproduction des algues et leurs rapports avec la phylogénie. Rev. Cytol., Paris, T. 13, pp. I-49.

FeldManN, J. \& G., I942. Recherches sur les Bonnemaisoniacées et leur alternance de générations. Ann. Sci. nat. (Bot.), Ire Série,'T. 3, pp. 75-175. 
Feldmann, J. \& G., I952. Nouvelles recherches sur le cycle des Bonnemaisoniacées: le développement des Tétraspores du Falkenbergia rufolanosa (Harv.) Schmitz. Rev. gén. Bot., T. 59, pp. 313-23.

Feldmann-Mazoyer, G., I940. Recherches sur les Céramiacées de la Méditerranée Occidentale. 5 IO pp., Alger.

Frémy, P., 1934. Cyanophycées des côtes d'Europe. Mém. Soc. nat. Sci. Cherbourg, T. 4I, pp. I-235.

FritsCH, F. E., I945. The Structure and Reproduction of the Algae, Vol. 2. Cambridge.

GeItLeR, L., I932. Cyanophyceae. Rabenhorst's Kryptogamen-Flora, Bd. I4. Leipzig.

Hamel, G., I924. Floridées de France. I. Rev. Algol., T. I, pp. 278-92.

— I93I. Chlorophycées des côtes françaises (Fin). Rev. Algol., T. o: pp. 9-73. 193I-1939. Pheophycées de France. Paris.

Harvey, W. H., I846-I85I. Phycologia Britannica, Vols. I-4. London.

Hollenberg, G. J., I94I. Observations on Ralfsiaceae. Amer. Fourn. Bot., Vol. 28, p. 728.

JAASUND, E., I951. Marine algae from northern Norway. I. Bot. Notiser, pp. I28-42.

Kolderup Rosenvinge, L., I924. The marine algae of Denmark. Pt. III. Rhodophyceae. III (Ceramiales). K. danske vidensk. Selsk., Ser. Nat., Math. Afd., 7, pp. $287-486$.

Kolderup Rosenvinge, L., with contributions by SÖRen Lund, 1935. On some Danish Phaeophyceae. K. danske vidensk. Selsk., Nat. Math. Afd., 9, Ser. vi, pp. I-40.

Kolderup Rosenvinge, L. ( $\dagger$ ) \& Lund, Sören, I947. The marine algae of Denmark. Vol. II. Phaeophyceae. III. Biol. Skr., Bd. 4, pp. I-99.

Kornmann, P., I953. Der Formenkreis von Acinetospora crinita (Carm.) nov.comb. Helgoländ. Wiss. Meeresunters., Bd. 4, pp. 205-24.

KosteR, J. Th., I952. Rare or otherwise interesting marine algae from the Netherlands. Acta Bot. Néerland., Vol. I, pp. 20I-I5.

KuCKUCK, P. ( $\dagger$ ), I953. (Ed. by P. Kornmann.) Ectocarpaceen-Studien. I. Hecatonema, Chilionema, Compsonema. Helgoländ. Wiss. Meeresunters., Bd. 4, pp. $316-52$.

KYLIN, H., I933. Über die Entwicklungsgeschichte der Phaeophyceen. K. fysiogr. Sällsk. Lund Förh., Bd. 44, I02 pp.

— 1934. Bemerkungen über einige Nitophyllaceen. K. fysiogr. Sällsk. Lund Förh., Bd. 4 , pp. I-8.

- 1935. Über einige kalkbohrende Chlorophyceen. K. fysiogr. Sällsk. Lund Förh., Bd. 5, I9 pp.

— 1944. Die Rhodophyceen der schwedischen westküste. K. fysiogr. Sällsk. Lund Förh., N.F., Bd. 55, I04 pp.

— I947. Die Phaeophyceen der schwedischen westküste. K. fysiogr. Sällsk. Lund Förh., N.F., Bd. 58, 99 pp.

— 1949. Die Chlorophyceen der schwedischen westküste. K. fysiogr. Sällsk. Lund Förh., N.F., Bd. 60, 79 pp.

Lanjouw, J. (ed. by), I952. International Code of Botanical Nomenclature adopted by the Seventh International Botanical Congress, Stockholm, Fuly I950, Utrecht.

Lemorne, Mme P., I928. Sur la présence de Lithophyllum orbiculatum Fosl. dans la Manche et son attribution au genre Pseudolithophyllum. Rev. Algol., T. 4, pp. I-6.

LeVRING, T., I937. Zur Kenntnis der Algenflora der norwegischen Westküste. K. fysiogr. Sällsk. Lund Förh., N.F., Bd. 48, I47 pp. 
LeVring, T., I940. Studien über die Algenvegetation von Blekinge, Südschweden. I 78 pp., Lund.

- 1945. Zur Kenntnis zweier endophytischer Phaeophyceen. Acta Hort. gothoburg., Bd. I6, pp. 185-90.

Lindstedt, A., 1943. Die Flora der marinen Cyanophyceen der schwedischen Westküste. I2I pp., Lund.

LuCAS, J. A. W., I950. The algae transported on drifting objects and washed ashore on the Netherlands' coast. Blumea, Vol. 6, pp. 527-43.

Lund, S., I950. The marine algae of Denmark. Vol. II. Phaeophyceae. IV. Biol. Skr., Bd. 6, pp. I-80.

Lyle, L., 1920. The marine algae of Guernsey. Fourn. Bot., Lond., Vol. 58, suppl. 2, $53 \mathrm{pp}$.

I923. Distribution of the marine flora of the Channel Islands compared with that of the coasts of Western Europe. Fourn. Ecol., Vol. I I, pp. 77-92.

Marshall, S. M., Newton, L., OrR, A. P. et al., I949. A Study of certain British Seaweeds and their Utilization in the Preparation of Agar. 184 pp. London: H.M. Stationery Office.

Nadson, G. A., I932. Contribution à l'étude des algues perforantes. Bull. Acad. Sci. U.R.S.S., vire Sér., no. 7 , pp. 833-45.

Newton, L., I93I. A Handbook of British Seaweeds. London.

PAPENFuss, G. F., 1950. Review of the genera of algae described by Stackhouse. Hydrobiologia, Vol. 2, pp. 181-208.

SchotTer, G., I952. Note sur le Gymnogongrus nicaeensis (Dub.) Ardiss. et Straff. Bull. Soct. Hist. nat. Afr. N., T. 43, pp. 203-10.

Setchell, W. A. \& Gardner, N. L., I925. The marine algae of the Pacific coast of North America. Pt. III. Melanophyceae. Univ. Calif. Publ. Bot., Vol. 8, pp. $383-898$.

SILVA, P. C., I 952 . A review of nomenclatural conservation in the algae from the point of view of the type method. Univ. Calif. Publ. Bot., Vol. 25, pp. 24I-324.

Sinclair, J., I949. The marine algae of Stronsay. Notes R. Bot. Gdn. Edinb. Vol. 20, pp. 160-79.

Smith, G. M., I950. The Fresh-water Algae of the United States. 2nd ed. London.

Veldkamp, H., I950. The genus Polysiphonia in the Netherlands. Blumea, Vol. 6, pp. $517-26$.

WAERN, M., I945. Remarks on some Swedish Sphacelariaceae. Svensk. bot. Tidskr., Bd. 39 , pp. $396-418$.

— I952. Rocky-shore algae in the Öregrund Archipelago. Acta phytogeogr. suec., Bd. 30, 298 pp. 